\title{
Atrofia muscular espinhal: possíveis impactos do rastreio precoce no teste do pezinho ampliado
}

\author{
Spinal muscular atrophy: possible impacts of early screening on the extended heel prick \\ test
}
Atrofia muscular espinal: posibles impactos de la detección temprana de la prueba de cribado neonatal

Graziela Cesar de Sousa ${ }^{1 *}$, Bruna de Oliveira Almeida ${ }^{2}$, Gyovana Rosa Vilela ${ }^{1}$, Juliana Marques Santos Ferreira ${ }^{1}$, Letícia Almeida Honorato ${ }^{1}$, Maria Caroline Castro Santos ${ }^{1}$, Mariana Cristina Flores Emiliano ${ }^{1}$, Roberta Ellen Santos Oliveira1', Patrícia Regina Guimarães ${ }^{1}$.

\section{RESUMO}

Objetivo: Apresentar aspectos importantes da Atrofia Muscular Espinhal (AME) e analisar os possíveis impactos do rastreio precoce através do Teste do Pezinho Ampliado no prognóstico das crianças acometidas. Revisão bibliográfica: AME é uma doença neuromuscular caracterizada pela degeneração de neurônios alfa na medula espinhal decorrente de uma deleção em 5q11.2-q13.3 que afeta o neurônio motor de sobrevivência 1 (SMN1). Cursa com atrofia muscular progressiva, fraqueza, paralisia e morte. A classificação tradicional divide a AME em 5 tipos, mas sua importância prática vem sendo progressivamente reduzida. A suspeição diagnóstica se dá pelas manifestações características da doença e a confirmação é feita por meio de testes genéticos moleculares. O manejo do paciente com AME envolve medidas não farmacológicas, além de terapia medicamentosa. A triagem neonatal objetiva rastrear doenças em recém-nascidos no período présintomático. Seis doenças estão incluídas no rastreio, mas outras serão incorporadas, incluindo a AME. Considerações finais: Como o sucesso da terapia está associado à precocidade de sua instituição, o rastreio precoce possibilitará melhor perspectiva de cuidado na AME, maximizando os resultados funcionais e promovendo qualidade de vida.

Palavras-chave: Atrofia muscular espinal, Atrofias musculares espinais da infância, Triagem neonatal.

\begin{abstract}
Objective: To present important aspects of Spinal Muscular Atrophy (SMA) and analyze the possible impacts of early screening using the Extended Pezinho Test on the prognosis of affected children. Bibliographic review: $A M E$ is a neuromuscular disease characterized by the degeneration of alpha neurons in the spinal cord resulting from a deletion in 5q11.2-q13.3 that affects the survival motor neuron 1 (SMN1). It courses with progressive muscle atrophy, weakness, paralysis and death. The traditional classification divides AME into 5 types, but its practical importance has been progressively reduced. The diagnostic suspicion is based on the characteristic manifestations of the disease and confirmation is made through molecular genetic tests. The management of patients with SMA involves non-pharmacological measures, in addition to drug therapy. Neonatal screening aims to screen for diseases in newborns in the pre-symptomatic period. Six diseases are included in the screening, but others will be incorporated, including AME. Final considerations: As the success of therapy is associated with the precocity of your institution, early screening will enable a better perspective of care in EBF, maximizing functional results and promoting quality of life.
\end{abstract}

Keywords: Spinal muscular atrophy, Spinal muscular atrophies of childhood, Neonatal screening.

1 Pontifícia Universidade Católica de Minas Gerais (PUC Minas), Betim - MG.

*E-mail: graziela.cesar@sga.pucminas.br

2 Faculdade Ciências Médicas de Minas Gerais, Belo Horizonte - MG. 


\section{RESUMEN}

Objetivo: Presentar aspectos importantes de la Atrofia Muscular Espinal (AME) y analizar los posibles impactos del cribado precoz mediante el Test de Pezinho Extendido en el pronóstico de los niños afectados. Revisión bibliográfica: AME es una enfermedad neuromuscular caracterizada por la degeneración de neuronas alfa en la médula espinal como resultado de una deleción en 5q11.2-q13.3 que afecta la neurona motora de supervivencia 1 (SMN1). Cursa con atrofia muscular progresiva, debilidad, parálisis y muerte. La clasificación tradicional divide AME en 5 tipos, pero su importancia práctica se ha ido reduciendo progresivamente. La sospecha diagnóstica se basa en las manifestaciones características de la enfermedad y la confirmación se realiza mediante pruebas genéticas moleculares. El manejo de pacientes con AME implica medidas no farmacológicas, además de la terapia con medicamentos. El cribado neonatal tiene como objetivo detectar enfermedades en los recién nacidos en el período presintomático. Se incluyen seis enfermedades en el cribado, pero se incorporarán otras, incluida la AME. Consideraciones finales: Dado que el éxito de la terapia está asociado con la precocidad de su institución, la detección temprana permitirá una mejor perspectiva de la atención en la LME, maximizando los resultados funcionales y promoviendo la calidad de vida.

Palabras clave: Atrofia muscular espinal, Atrofias musculares espinales de la infancia, Cribado neonatal.

\section{INTRODUÇÃO}

A triagem neonatal foi instituída como política pública de oferta obrigatória e gratuita em 1992. A coleta da amostra de sangue do calcâneo do recém-nascido é preferencialmente realizada entre o $3^{\circ}$ e $5^{\circ}$ dia de vida e permite a triagem de seis doenças: fenilcetonúria, hipotireoidismo congênito, deficiência da biotinidase, fibrose cística, anemia falciforme e hiperplasia adrenal congênita. Em maio de 2021, foi sancionada a lei federal que ampliou o espectro de doenças rastreadas na triagem neonatal com cobertura do Sistema Único de Saúde (SUS) para cinquenta doenças, entre elas, a Atrofia Muscular Espinhal (AME), cuja inclusão será feita na última das cinco etapas de implementação do Teste do Pezinho Ampliado (SOCIEDADE BRASILEIRA DE PEDIATRIA, 2021).

A AME é uma doença genética de herança autossômica recessiva que ocorre, mais comumente, por deleção total ou parcial do gene do neurônio motor de sobrevivência 1 (SMN1), localizado no cromossomo 5q. A deleção impossibilita a síntese da proteína SMN, essencial para o funcionamento dos neurônios motores, fazendo com que atividades essenciais, como marcha, fala, deglutição e respiração fiquem comprometidas. É uma doença de evolução progressiva com incidência de 1 para 6.000 até 1 para 11.000 nascidos vivos. Dentre as condições monogênicas, a AME é a que mais frequentemente culmina no óbito infantil (MINISTÉRIO DA SAÚDE, 2019).

A suspeita e consequente investigação para AME ocorrem a partir de sinais e sintomas clínicos típicos do acometimento neuromuscular. Em crianças com AME tipo I sem tratamento, ocorre degeneração de $95 \%$ dos neurônios motores espinhais até os 6 meses de idade, reduzindo drasticamente a qualidade de vida e a funcionalidade destes pacientes. O teste genético molecular é o padrão ouro para diagnóstico de AME, embora outros métodos também possam ser empregados, quando necessário (MERCURI E, et al., 2018).

O manejo dos pacientes com AME envolve medidas não farmacológicas que objetivam maximizar o desenvolvimento neuropsicomotor e promover suporte. Entre as possibilidades terapêuticas estão o suporte respiratório, o acompanhamento ortopédico, nutricional e fisioterápico (MERCURI E, et al., 2018). Há também uma perspectiva promissora de tratamento farmacológico baseada na administração do medicamento Nusinersen (Spinraza ${ }^{\mathrm{TM}}$ ) que obteve excelentes resultados nos testes clínicos, sendo capaz de impedir a progressão da destruição neuronal, possibilitando que as crianças atinjam os marcos motores do desenvolvimento e obtenham melhor qualidade de vida (FINKEL RS, et. al., 2018).

Considerando que o sucesso do tratamento está diretamente relacionado ao período de instituição da terapêutica e que resultados mais expressivos são obtidos com a instituição precoce, este trabalho teve como objetivo apresentar os possíveis impactos do rastreio precoce da AME no prognóstico das crianças acometidas. 


\section{REVISÃO BIBLIOGRÁFICA}

\section{Atrofia Muscular Espinhal: classificação e manifestações clínicas}

Designam-se como Atrofias Musculares Espinhais as doenças neuromusculares cuja etiopatogenia envolve a degeneração de neurônios alfa na medula espinhal. O espectro denominado AME consiste no tipo mais comum de atrofia muscular espinhal e tal como as demais, cursa com atrofia muscular progressiva e fraqueza, podendo levar à paralisia parcial ou total (BURNS JK, et al., 2016; GLASCOCK J, et al., 2018; MERCURI E, et al., 2018). A AME representa importante causa de óbito infantil e atualmente é incurável (BURNS JK, et al., 2016; GLASCOCK J, et al., 2018).

Os indivíduos acometidos por AME apresentam sinais clínicos característicos cuja intensidade varia de acordo com o comprometimento neuromuscular. Os principais sinais são a hipotonia e a fraqueza que afeta mais os membros inferiores do que os superiores e se manifesta de forma semelhante bilateralmente. Músculos envolvidos na dinâmica respiratória são frequentemente acometidos pela fraqueza muscular, predispondo os pacientes à necessidade de suporte ventilatório. Na maior parte das vezes, são estes sinais que promovem a suspeição diagnóstica, já que a doença tem uma fase pré-sintomática variável e não há investigação de rotina na ausência de história familiar positiva (MINISTÉRIO DA SAÚDE, 2019). Sinais que surgem muito precocemente são indícios de evolução mais grave e prognóstico reservado (BURNS JK, et al., 2016).

A ocorrência de AME é dependente de uma deleção genética em 5q11.2-q13.3 do gene SMN1 que impede a formação da proteína SMN funcional. Os indivíduos acometidos pela deleção apresentam um gene parálogo, o SMN2, que apresenta uma produção ligeiramente menor, porém essencial, de proteínas funcionantes. A determinação do número de cópias de SMN2 é uma maneira fidedigna de estimar a gravidade do acometimento, sendo esta tanto maior quanto menor o número de cópias (DE VIVO DC, et al., 2019). É importante ressaltar, no entanto, que esta relação não é absoluta e pode não corresponder às expectativas de prognóstico (MERCURI E et al., 2018).

A AME é classicamente dividida em 5 tipos cuja distinção é feita basicamente analisando a precocidade das manifestações clínicas, a gravidade dos sinais e sintomas e as habilidades neuropsicomotoras desenvolvidas (MINISTÉRIO DA SAÚDE, 2019).

O tipo 0 é o que apresenta pior prognóstico e geralmente está relacionado com a presença de uma única cópia do gene SMN2. Os sinais podem se manifestar ainda intraútero através de redução importante da movimentação fetal. As crianças com AME tipo 0 , também conhecida como tipo $1 \mathrm{~A}$, se apresentam com hipotonia generalizada ao nascimento e insuficiência respiratória grave, raramente sobrevivendo ao primeiro mês pós-natal (BURNS JK, et al., 2016;).

O tipo 1, também chamado de doença de Werdnig-Hoffmann, representa até $60 \%$ dos casos de AME. Manifesta-se até o sexto mês de vida e é caracterizado por hipotonia significativa, fraqueza muscular e necessidade de suporte ventilatório antes do primeiro ano de vida. A criança não consegue sustentar o pescoço ou sentar sem apoio e apresenta engasgos frequentes por uma dificuldade de deglutição inerente à degeneração neuromuscular. Este tipo, geralmente, está relacionado à presença de duas cópias do gene SMN2, embora a classificação seja suportada unicamente por parâmetros clínicos e não pela análise genética (MERCURI E, et al., 2018).

No tipo 2, as manifestações clínicas surgem entre os seis e os 18 meses de idade. As crianças apresentam fraqueza muscular, dificuldade respiratória e alterações na deglutição em níveis variados, embora menos importantes que na AME tipo 1. A criança é capaz de sentar sem apoio, mas não consegue deambular. É comum que, com a evolução da doença e o crescimento da criança, surjam déficits posturais e osteomusculares principalmente caracterizados por escoliose, deslocamento de quadril e deformidades articulares. O tipo 2 responde por cerca de $30 \%$ dos casos de AME e, geralmente, há três cópias do gene SMN2 (GLASCOCK J, et al., 2018).

No tipo 3, também chamado de Kugelberg-Welander, os sinais se iniciam após os 18 meses de idade. À esta altura, as crianças já aprenderam a se sustentar sem apoio e a caminhar. No entanto, ao longo do desenvolvimento, podem perder essas habilidades e necessitar de cadeira de rodas. A fraqueza muscular é 
um sinal característico e acomete de forma mais importante os membros inferiores do que os superiores. Podem ocorrer alterações da anatomia da coluna vertebral, embora mais raramente do que as identificadas no tipo 2. Este tipo corresponde a cerca de 10\% dos diagnósticos de AME (MERCURI E, et al., 2018).

O tipo 4 é mais raro e apresenta manifestações clínicas mais brandas que se iniciam, normalmente, na vida adulta. Os indivíduos adquirem a capacidade de deambular e não a perdem, mas apresentam fraqueza importante nos membros inferiores que podem provocar alguma dificuldade na marcha. Esse tipo compreende apenas uma pequena parcela dos pacientes com AME (BURNS JK, et al., 2016).

Considerando as alternativas recentes para intervir na história natural da doença, essa classificação tem se tornado cada vez menos aplicável, já que um número crescente de crianças tratadas consegue atingir e manter marcos do desenvolvimento que não seriam esperados considerando o tipo de AME que possuem. Assim, a classificação mais útil atualmente consiste na separação entre indivíduos que não sentam (ou "nonsitters"); indivíduos que sentam (ou "sitters"); e indivíduos que andam (ou "walkers") (MERCURI E, et al., 2018)

\section{Diagnóstico da Atrofia Muscular Espinhal}

A suspeita clínica de AME baseia-se na observação das manifestações clínicas características da doença, como hipotonia, fraqueza progressiva simétrica e proximal que poupa os músculos faciais e acomete de maneira mais importante as pernas do que os braços. A confirmação diagnóstica da doença se dá por testes genéticos moleculares. O teste genético de SMN1/SMN2 é altamente confiável e consiste na investigação de primeira linha diante de quadro clínico típico. O padrão ouro consiste na análise quantitativa dos genes SMN1 e SMN2 por meio de três principais métodos: MLPA (multiplex ligation-dependent probe amplification), qPCR (quantitative polymerase chain reaction) e sequenciamento de próxima geração (Next Generation Sequencing -NGS). As apresentações mais características da doença dispensam a realização de biópsia muscular. A eletromiografia pode ser necessária em formas crônicas, em que o fenótipo é menos marcante, sendo geralmente desnecessária nos tipos 1 e 2 (MERCURI E, et al., 2018)

O diagnóstico de AME é confirmado porque esses pacientes apresentam perda homozigótica da função de ambas as cópias de SMN1. Quando uma das cópias foi deletada e o paciente apresenta manifestações típicas da doença, deve-se sequenciar a cópia presente para investigar a existência de mutações mais sutis que impeçam a formação adequada das proteínas. A presença de duas cópias completas do SMN1 torna improvável o diagnóstico de AME, mas o sequenciamento dos genes continua sendo válido em casos clínicos muito sugestivos. Para estes pacientes é importante considerar e investigar diagnósticos diferenciais importantes, principalmente acometimentos dos neurônios motores. A deleção homozigótica de SMN1 apresenta $100 \%$ de especificidade para o diagnóstico, sendo a gravidade da doença modificada pelo número de cópias de SMN2. A maioria dos indivíduos saudáveis na população apresenta 0-3 cópias de SMN2 (ARNOLD WD, et al., 2014)

\section{Tratamento não-farmacológico}

A AME é um transtorno complexo e que desencadeia inúmeras complicações, muitas passíveis de suporte. O tratamento respiratório com suporte de pressão positiva nas vias aéreas quando propício, o manejo ortopédico da escoliose e outras deformidades, o suporte nutricional e o acompanhamento fisioterápico têm apresentado diferenças expressivas nos resultados clínicos (GLASCOCK J, et al., 2018).

O exame físico dos pacientes com AME deve ser focado no sistema músculo esquelético e deficiências funcionais relacionadas. Esse exame deve ser realizado a cada seis meses por examinadores treinados. As avaliações são realizadas de forma diferente para cada nível de gravidade ("non-sitters", "sitters" ou "walkers") e incluem avaliação de força e amplitude de movimento articular, escalas funcionais motoras e testes cronometrados para monitorar os aspectos da função que refletem as atividades de vida diária (MERCURI E, et al., 2018).

Em relação aos cuidados ortopédicos, condutas são destinadas afim de evitar fraturas, manter os movimentos e tratar deformidades, visto que a escoliose, a cifose torácica e deformidades do tórax são muito comuns em pacientes com atrofia muscular espinhal (MINISTÉRIO DA SAÚDE, 2019). 
Evidências têm emergido a favor de uma abordagem proativa, incluindo sessões regulares de fisioterapia. Os objetivos da reabilitação diferem conforme a gravidade. Com relação aos que não sentam, os objetivos são otimização da função, minimização da deficiência e otimização da tolerância a várias posições. Já em relação aos que sentam, pretende-se prevenir contraturas e escolioses e manter, restaurar ou promover a função e a mobilidade. Ambos irão trabalhar com alongamento, posicionamento, fisioterapia torácica, mobilidade e exercício. Por sua vez, os principais objetivos da reabilitação para os pacientes caminhantes são manter, restaurar ou promover a função, a mobilidade e a amplitude articular adequada, além de melhorar o equilíbrio e a resistência (MERCURI E, et al., 2018).

É necessário que se faça uma avaliação respiratória frequente, pois é comum que os pacientes com AME $5 q$ tipo I apresentem um diafragma relativamente forte e músculos intercostais fracos, aliado a dificuldade de tossir e eliminar muco, o que pode resultar em hipoventilação, atelectasia, depuração deficiente das secreções das vias aéreas e infecções recorrentes. Assim, a fisioterapia respiratória é essencial nos cuidados desses pacientes. Em alguns casos a ventilação mecânica não invasiva (VNI) e a ventilação mecânica são indicados (MINISTÉRIO DA SAÚDE, 2019).

O suporte nutricional e a avaliação do crescimento são importantes em todos os tipos de AME. A consulta nutricional é imprescindível para manter uma dieta adequada, tanto em relação ao controle de peso, quanto à ingestão de macro e micronutrientes, em especial cálcio e vitamina $D$ para a saúde óssea. A investigação de sintomas gastrointestinais é importante em todos os casos. Em "non-sitters" e "sitters" é muito importante considerar a deglutição segura e o risco de aspiração. Na população dos caminhantes, a disfunção de deglutição e a dificuldade da alimentação são raras, o que se torna mais preocupante é a obesidade e o sobrepeso, pois são fatores dificultadores da locomoção (MERCURI E, et al., 2018).

Atualmente, é sugerido que esse manejo multidisciplinar seja coordenado por um médico, principalmente neurologista/ neurologista pediátrico, para monitorização da progressão e implementação de medidas antecipatórias, quando possível. Dessa forma, os doentes devem ser avaliados e tratados conforme os locais de manifestação da doença, visto que o acompanhamento longitudinal e os cuidados paliativos contribuem para uma melhor evolução da doença, além de aumentar a sobrevida (MINISTÉRIO DA SAÚDE, 2019).

\section{Tratamento farmacológico}

O tratamento farmacológico proposto para AME ainda carece de evidências em relação à eficácia e à segurança. O único medicamento que completou os ensaios clínicos de fase 3 para AME tipo 1 e tipo 2 é o nusinersen (Spinraza ${ }^{\mathrm{TM}}$ ), o qual obteve aprovação nos Estados Unidos pela Food and Drug Administration (FDA) e na Europa pela European Medicines Agency (EMA) (FINKEL RS, et al., 2018).

O nusinersen é um oligonucleotídeo anti-sense administrado por via intratecal, que apresentou benefício relevante e clinicamente significativo como recurso terapêutico para a AME (DE VIVO DC, et al., 2019). Revisão sistemática que avaliou a eficácia do nusinersen revelou que, se iniciado antes do início dos sintomas (período pré-sintomático), há melhora da sobrevida e do desenvolvimento motor. Para crianças com AME tipo 1, o tratamento com nusinersen demonstrou melhora da sobrevida sem suporte ventilatório permanente e do desenvolvimento da função motora. Para crianças com AME tipos 2 e 3, a terapêutica resultou em um melhor desenvolvimento ou estabilização da função motora. É importante frisar que o tratamento demonstrou ser mais eficaz quanto mais cedo for iniciado e quanto menor a duração da doença. No entanto, a estrutura institucional necessária para a administração e o monitoramento pós-procedimento e o custo do medicamento são fatores limitantes (ALBRECHTSEN SS, et al., 2020).

O albuterol, um agonista beta-adrenérgico, é utilizado frequentemente na prática clínica em alguns países, apesar da falta de estudos randomizados controlados por placebo. Quanto ao uso de antibióticos, suplementos para a saúde óssea (como vitamina $D$, cálcio e bisfosfonato) e medicamentos para refluxo gastroesofágico, a recomendação existe, com exceção da vitamina D. Entretanto, a profilaxia com tais terapêuticas é rara, sendo utilizadas principalmente em caso de deficiências comprovadas (FINKEL RS, et al., 2018). 


\section{A triagem neonatal ampliada}

A triagem neonatal, popularmente conhecida como "teste do pezinho", é um programa de saúde pública que integra um conjunto de ações com o objetivo de detectar precocemente recém-nascidos com doenças metabólicas, genéticas, enzimáticas e endocrinológicas. Tem como objetivo garantir uma intervenção adequada em tempo hábil, bem como o melhor tratamento, evitando sequelas e até mesmo a morte. $O$ teste do pezinho deve ser realizado, preferencialmente, entre $\circ 3^{\circ}$ e $\circ 5^{\circ}$ dia de vida do recém-nascido e consiste na coleta e análise de uma gota de sangue do calcanhar do bebê em cartão de coleta com papel-filtro (MINISTÉRIO DA SAÚDE, 2016; OLIVEIRA JCDA, et al., 2021).

Desde 1960, a Organização Mundial de Saúde (OMS) preconiza a realização da triagem neonatal para a prevenção de deficiência mental e agravos à saúde do recém-nascido, sobretudo, em países em desenvolvimento. No entanto, a triagem neonatal só foi incorporada ao Sistema Único de Saúde (SUS), no ano de 1992, com a obrigatoriedade de realização do teste que incluía o rastreio de fenilcetonúria e hipotireoidismo congênito (BAGGIO FL, et al., 2020; FERNANDES MSSN e CARDOSO AM, 2018).

Em 2001, o Ministério da Saúde, por meio da Secretaria de Assistência à Saúde, reavaliou a triagem neonatal no SUS, o que culminou na sua reestruturação e criação do Programa Nacional de Triagem Neonatal (PNTN), com o objetivo de ampliação da política. Assim, a partir deste ano, foram incluídas a triagem de hemoglobinopatias; deficiência da biotinidase; fenilcetonúria; hipotireoidismo congênito; fibrose cística; e hiperplasia adrenal congênita (BAGGIO FL, et al., 2020).

Em 2015 foi aprovado o projeto que obriga o SUS a ampliar periodicamente o rol de exames feitos em recém-nascidos para detectar doenças, pois apenas seis grupos são alvo das políticas brasileiras de rastreamento, enquanto outros países como França, Inglaterra e Alemanha triam até 14 tipos de doenças, e nos Estados Unidos, o exame chega a englobar 53 diagnósticos. Dessa forma, para aprimorar o PNTN, em 26 de maio de 2021 foi sancionada a lei federal que amplia de seis para 53 o número de doenças detectadas pelo teste do pezinho, entre as quais está a AME (AGÊNCIA SENADO, 2015; CASTRO REVD, 2021).

A ampliação da triagem neonatal ocorrerá gradualmente, seguindo cinco etapas, cuja primeira incluirá ao rol de doenças triadas a toxoplasmose congênita, hemoglobinopatias e hiperfenilalaninemias. Na segunda etapa, serão incluídos os rastreios de galactosemias, aminoacidopatias, distúrbios do ciclo da ureia e distúrbios da beta oxidação dos ácidos graxos. Na terceira etapa, serão incluídas as doenças lisossômicas. $\mathrm{Na}$ quarta etapa, as imunodeficiências primárias. Por fim, a AME será incluída na quinta etapa (CASTRO REVD, 2021).

A ampliação da triagem neonatal poderá garantir melhores condições de saúde para todas as crianças brasileiras, uma vez que vai diagnosticar doenças congênitas de manifestação precoce e evolução para complicações clínicas permanentes e extremamente graves, contribuindo assim, para a redução da mortalidade infantil no país (SOCIEDADE BRASILEIRA DE PEDIATRIA, 2021).

\section{Impactos do diagnóstico precoce no prognóstico da Atrofia Muscular Espinhal}

O prognóstico da AME está intimamente ligado à história natural da doença, que vem se modificando e se atualizando drasticamente devido ao avanço dos tratamentos disponíveis. A perda rápida e irreversível de neurônios motores começa geralmente até os 3 meses de idade, cerca de $95 \%$ dos neurônios motores são perdidos aos 6 meses e o progresso para insuficiência respiratória e morte acontece antes dos 2 anos (YINHSIU CHIEN MD, et al., 2017).

A classificação da AME baseada na idade de início das perdas das capacidades motoras está sendo discutida devido às alterações da história natural da doença percebidas nos pacientes submetidos ao tratamento em momento oportuno. Pacientes que conquistam marcos em etapas não previstas acabam se tornando um desafio de classificação, quando são utilizados os critérios originais (JEDRZEJOWSKA M, 2020).

Sendo assim, o prognóstico da Atrofia Muscular Espinhal está diretamente relacionado a dois fatores: a deficiência de base da proteína SMN e o momento de instituição da terapia. Tendo em vista os promissores resultados obtidos com fármacos direcionados ao tratamento da doença e os melhores resultados associados à administração precoce, o diagnóstico em tempo oportuno é parte essencial do manejo destes pacientes (FARRAR MA, et al., 2017). 
O sucesso do tratamento para a doença depende fortemente da idade do paciente no momento da terapia e no tempo de duração da doença. Quanto mais novo for o paciente e menor for a duração da doença, melhor é o prognóstico. A terapia idealmente deve ser instituída quando os neurônios motores ainda estão viáveis e quando a regulação positiva da proteína SMN ainda está disponível para recriar os processos fisiológicos. Essa ideia é traduzida como: "tempo é neurônio motor" (VILL K, 2019).

Em casos graves e extremamente graves a deficiência de SMN acontece no período pré-natal e por isso não é possível restaurar completamente as funções motoras após o nascimento da criança. Por outro lado, para os casos moderados a janela terapêutica ainda não é bem definida. De qualquer forma, a grande maioria dos pacientes irão se beneficiar com o diagnóstico, e consequente tratamento, precoces (YIN-HSIU CHIEN MD, et al., 2017).

O Spinraza (Nusisersen), o primeiro e mais popular medicamento instituído para tratamento de crianças com AME obteve resultados promissores documentados pelo ensaio clínico NURTURE. Após um seguimento de 2,9 anos de 25 pacientes com 2 ou 3 cópias do SMN 2 que receberam o medicamento no período présintomático, $100 \%$ das crianças estavam vivas, $0 \%$ em ventilação definitiva, $100 \%$ atingiram a habilidade de sentar sozinhas e $88 \%$ conquistaram a habilidade de andar sozinhas. Os resultados foram mais significativos nas crianças que receberam o tratamento antes dos 5 meses quando comparadas com as que receberam entre 5 e 7 meses de idade (JEDRZEJOWSKA M, 2020).

\section{CONSIDERAÇÕES FINAIS}

A suspeição do diagnóstico da AME é feita a partir de manifestações clínicas típicas que ocorrem após a degeneração irreparável dos neurônios motores. Uma intervenção que possibilite alteração na história natural da doença e, consequentemente, melhore o prognóstico dos pacientes, depende, necessariamente, da identificação precoce e da instituição de terapêutica em tempo oportuno. A expectativa com a ampliação do Teste do Pezinho e com a inclusão do rastreio da AME é de que a intervenção no período pré-sintomático possibilite a preservação dos neurônios, otimize as medidas terapêuticas e o alcance de marcos importantes do desenvolvimento neuropsicomotor, garantindo maior funcionalidade e qualidade de vida para estas crianças.

\section{REFERÊNCIAS}

1. AGÊNCIA SENADO. 2015. Teste do pezinho pode ser ampliado. Disponível em: https://www12.senado.leg.br/noticias/materias/2015/09/09/teste-do-pezinho-pode-ser-ampliado. Acesso em: 19 jun. 2021.

2. ALATORRE-JIMÉNEZ MA, et al. Spinal muscular atrophy: review of a child onset disease. Journal of Advances in Medicine and Medical Research, 2015; 7(6): 647-660.

3. ALBRECHTSEN SS, et al. Nusinersen treatment of spinal muscular atrophy - a systematic review. Dan Med J, 2020; 67(9): A02200100.

4. BAGGIO FL, et al. Produção de conhecimento sobre as doenças rastreadas pela triagem neonatal no Brasil de 2008 a 2018. Revista Eletrônica Acervo Saúde, 2020; 45: 2596-2596.

5. BURNS JK, et al. Opening the window: The case for carrier and perinatal screening for spinal muscular atrophy. Neuromuscular disorders, 2016; 26(9): 551-559.

6. CASTRO REVD. PEBMED. 2021. Sancionada lei para ampliação do teste do pezinho pelo Sistema Único de Saúde (SUS). Disponível em: https://pebmed.com.br/sancionada-lei-para-ampliacao-do-teste-do-pezinho-pelo-sus/. Acesso em: 19 jun. 2021.

7. CHIEN YH, et al. Presymptomatic Diagnosis of Spinal Muscular Atrophy Through Newborn Screening. The Journal of Pediatrics, 2017;190:124-129.

8. DE VIVO DC, et al. Nusinersen initiated in infants during the presymptomatic stage of spinal muscular atrophy: Interim efficacy and safety results from the Phase 2 NURTURE study. Neuromuscular disorders, 2019; 29(11): 842-856.

9. FERNANDES MSSN, CARDOSO AM. Educação em saúde como estratégia da enfermagem na disseminação de informações sobre a triagem neonatal às futuras mães. Revista científica da escola estadual de saúde pública de Goiás, 2018; 4(1): 074-080.

10. FERRARI ET. Atrofia muscular espinal infantil. Protocolos diagnósticos y terapéuticos en Pediatría. 2010; 1: 125-130

11. FIKEL RS, et al. Diagnosis and management of spinal muscular atrophy: Part 2: Pulmonary and acute care; medications, supplements and immunizations; other organ systems; and ethics. Neuromuscular Disorders, 2018; 28(3): 197-207. 
12. GLASCOCK J, et al. Treatment Algorithm for Infants Diagnosed with Spinal Muscular Atrophy through Newborn Screening. Journal of Neuromuscular Diseases, 2018; 5(2): 145-158.

13. KRASZEWSKI JN, et al. Pilot study of population-based newborn screening for spinal muscular atrophy in New York state. Genetics in medicine: official journal of the American College of Medical Genetics. 2018; 20(6): 608-613.

14. MERCURI E, et al. Diagnosis and management of spinal muscular atrophy: Part 1: Recommendations for diagnosis, rehabilitation, orthopedic and nutritional care. Neuromuscular disorders, 2018; 28(2): 103-115.

15. MINISTÉRIO DA SAÚDE. Secretaria de Atenção à Saúde, Departamento de Atenção Especializada e Temática. Trigem Neonatal Biológica: Manual Técnico. Brasil, 2016. Disponível em: https://bvsms.saude.gov.br/bvs/publicacoes/triagem_neonatal_biologica_manual_tecnico.pdf. Acessado em: 19 de junho de 2021.

16. MINISTÉRIO DA SAÚDE. Secretaria de Atenção Especializada à Saúde. Portaria Conjunta № 15, de 22 de outubro de 2019. Aprova o Protocolo Clínico e Diretrizes Terapêuticas da Atrofia Muscular Espinhal 5q tipo I. Brasil, 2019. Disponível em: https://portalarquivos2.saude.gov.br/images/pdf/2019/outubro/23/Portaria-Conjunta-PCDT-AtrofiaMuscular-Espinhal-5q-Tipo-I-final.pdf. Acessado em: 10 ago. 2021.

17. MULLER-FELBER W, et al. Infants Diagnosed with Spinal Muscular Atrophy and 4 SMN2 Copies through Newborn Screening - Opportunity or Burden?. Journal of neuromuscular diseases, 2020; 7(2): 109-117.

18. OLIVEIRA JCDA, et al. Triagem Neonatal Ampliada. Brazilian Journal of Surgery and Clinical Research - BJSCR, $2021 ; 34: 61-64$.

19. ROSS LF, KWON JM. Spinal Muscular Atrophy: Past, Present, and Future. Neoreviews. 2019; 20(8): 437-451.

20. SOCIEDADE BRASILEIRA DE PEDIATRIA (SBP). SBP comemora aprovação do projeto de lei que regulamenta a aplicação do Teste do Pezinho Ampliado no SUS. Disponível em: https://www.sbp.com.br/imprensa/detalhe/nid/sbpcomemora-aprovacao-do-projeto-de-lei-que-regulamenta-a-aplicacao-do-teste-do-pezinho-ampliado-no-sus/. Acesso em: 19 jun. 2021.

21. STRUNK A, et al. Validation of a Fast, Robust, Inexpensive, Two-Tiered Neonatal Screening Test algorithm on Dried Blood Spots for Spinal Muscular Atrophy. International journal of neonatal screening, 2019; 5(2): 1-9.

22. VILL K, et al. Spinal muscular atrophy: Time for newborn screening?. Der Nervenarzt. 2017;88(12):1358-1366.

23. VILL K, et al. One Year of Newborn Screening for SMA - Results of a German Pilot Project. J Neuromuscul Dis. 2019;6(4):503-515. doi:10.3233/JND-190428

24. WENG WC, et al. CMAP changes upon symptom onset and during treatment in spinal muscular atrophy patients: lessons learned from newborn screening. Genetics in medicine: official journal of the American College of Medical Genetics, 2021; 23(2): 415-420. 